\title{
LXV. Observations upon the decomposition of the double cyanides by an electric current
}

\author{
Mr. James Napier
}

To cite this article: Mr. James Napier (1844) LXV. Observations upon the decomposition of the double cyanides by an electric current, Philosophical Magazine Series 3, 25:167, 379-382, DOI: $10.1080 / 14786444408645016$

To link to this article: http://dx.doi.org/10.1080/14786444408645016

曲 Published online: 30 Apr 2009.

Submit your article to this journal $[\pi$

Џ Article views: 2

Q View related articles $₫$ 


\section{[ 379 ]}

LXV. Observations upon the Decomposition of the Double Cyanides by an Electric Current. By Mr. James Napier*.

FOR some time past $I$ have considered that there were phæ-

nomena presenting themselves in my daily operations of depositing metals by a galvanic current of sufficient scientific interest to warrant their being collected and given in detail to this Society. In anticipation of doing so, I carefully noted down what seemed most interesting, in hopes of being prepared to give them as a short series of papers next session: I say series, because so far as I have observed, all the double cyanides, even when they have the same constitution, do not comport themselves in the same manner, giving rise to different results, all of which could not be given in the compass of one paper. But these anticipations have been somewhat changed by the circumstance, that a paper upon a similar subject has lately been read by Prof. Daniell to the Royal Society. The facts brought forward in that paper are as yet unknown to me, further than that certain metals are not transferred from one pole of the galvanic circuit to the other by means of the current; this is one of the facts to which my attention has been directed for a long time, and I am induced to bring this paper forward to-night, not with any desire of sharing the honours due to the discovery, but in hopes that, while noticing this in connection with other facts which I hope to have the honour of laying before you next session, I may have a little more claim upon your attention, from its having been original on my part.

The present paper is more particularly devoted to cyanide of potassium and silver, - a double salt of general use in electro-metallurgical operations, and one of a class eminently fitted to exhibit some of the most interesting facts in connection with electrical decompositions. It is known to those engaged in the deposition of silver from this salt, that if it be used pure, or in a neutral state, with a positive electrode of silver, no deposition of metal is obtained unless a battery of great power be used; but if a little cyanide of potassium be added to the solution, a very weak current of electricity is sufficient to give a deposit. The usual explanation of these facts is, that pure or neutral cyanide of potassium and silver is a bad conductor of electricity, and that the addition of free cyanide of potassium gives it conducting power, either as being the conducting medium, or by a kind of disposing influence which it imparts to the salt decomposed. That one fluid imparts such an

* Communicated by the Chemical Society; having been read May 6, 1844. 
influence to another I need hardly say is absurd; and it is well known that the conducting medium in any fluid is the substance decomposed. It is certain that the fiee cyanide of potassium, which is added with the idea of giving conduction, is not decomposed. The question then occurs, what part does it take in these operations? I have observed that, whilst endeavouring to deposit from the neutral salt, the positive electrode, although brightly polished previous to being put into the solution, instantly assumed a chalky aspect. It appeared probable that this was owing to the formation of cyanide of silver, which, being insoluble in the neutral salt, obstructed the current of electricity by its coating. To prove this, I placed a quantity of crystallized cyanide of potassium and silver, dissolved in water, in a long shallow vessel, kept the two electrodes at a considerable distance apart, and connected them with a battery of 20 pairs of plates for fortyeight hours: there was a considerable deposit of silver upon the cathode, the solution around it was strongly alkaline, and the precipitate formed by adding a drop of nitrate of silver was easily redissolved; whilst that part of the solution round the anode remained neutral, the anode was covered over with a grayish-white powder, which being washed exhibited every character of cyanide of silver. The use of the addition of free cyanide of potassium to the silver solution was now evident, not to facilitate the passage of the current, but to dissolve the cyanide of silver formed upon the electrode. To make this the more certain, I dissolved 2 equivalents of the cyanide of potassium, or twice 200 grains, and put them into a vessel divided by a porous diaphragm: into the division in which the anode was placed I put an equivalent of cyanide of potassium, or 66 grains; the electrodes being previously weighed, were connected with a battery of 4 pairs, deposition immediately took place; the current was continued for twelve hours; towards the end of the operation hydrogen gas began to be evolved from the cathode, and a powder to appear upon the anode; the experiment was then stopped; the anode being washed and weighed indicated a loss of 108.7 grains; the solution in which it was placed was neutral, and on being evaporated and fused yielded $214^{\circ} 3$ grains of silver; the cathode had gained 107.4 grains in weight; the solution in which it was placed had a powerful alkaline reaction, smelled strongly of hydrocyanic acid, and on being tested scarcely indicated the presence of silver. This last circumstance attracted particular attention, and induced me to repeat the experiment under various modifications, also with gold, copper and zinc, all of which gave similar results. 
Another question suggested itself in connection with the decomposition of cyanide of potassium and silver, namely, does the cyanide of potassium which is in union with the cyanide of silver take any part in these decompositions? To determine this, the experiment just detailed was repeated, taking care to reduce the power of the current of electricity when any indication of gas was perceived at the cathode; when the experiment was completed, the solution containing the negative electrode was tested for the quantity of cyanide of potassium by nitrate of silver; it indicated $67 \cdot 4$ grains, which is more than an equivalent, independent of loss by decomposition. The reason of the cyanide of potassium being more than the equivalent, is from that invariable endosmose which takes place when a current of electricity is passing which is in the direction of the negative division. From this experiment it appears that the cyanide of potassium which is combined with the cyanide of silver undergoes no change by the electric current, if it be properly regulated to the condition of the solution, so long as cyanide of silver is present: this exhibits beautifully the different conducting power of the two salts, and so long as there is not more electricity than the best conductor can transmit, it will pass through it in preference to any other salt mixed with it, although such other may be also an excellent conductor. But when the current is more than the best conductor will transmit, it passes through the next best present, which accordingly suffers decomposition; so that in the case of cyanide of potassium and silver, when the current is more than the cyanide of silver will transmit, the cyanide of potassium is also decomposed, so that two proportionals of cyanogen pass to the positive electrode, which being dissolved increases the quantity of silver in the solution, while potassium is liberated at the negative electrode, decomposing water with the escape of hydrogen gas.

If platinum electrodes be used there is no necessity for adding free cyanide of potassium, the pure solution being decomposed by a weak current, and the cyanngen liberated at the anode is absorbed by the solution, turning it dark brown and leaving a slight blackish precipitate, probably paracyanogen; this has been already observed by Professor Faraday in cyanide of potassium alone; but if the current be increased to 7 or 8 pairs of plates, oxygen gas is evolved from the anode, and cyanide of silver is precipitated upon its surface, the cyanogen being decomposed forming ammonia and probably formic acid, which latter decomposes the cyanide of potassium combined with the cyanide of silver, forming hydrocyanic acid and formiate of potash, the cyanide of silver being precipitated. Several other reactions take place, but my experiments 
are not yet sufficiently numerous to allow of my venturing upon a description of them in this paper.

It is evident, therefore, that the cyanide of potassium and silver in a neutral state is a most excellent conductor of electricity. A little cyanide of potassium added does however facilitate its decomposition; and it is remarkable that when the cyanide of potassium added is in such proportion as to form a compound having two equivalents of cyanide of potassium to one of cyanide of silver, it is probably the most easily decomposed substance that we have. I have deposited silver from it easily with one square inch of copper and zinc immersed in water, the solution being at a temperature of $75^{\circ}$.

Another question suggested itself in connection with this salt, namely, the manner in which the cyanogen is transferred from the negative to the positive electrode, whether it travels direct, or is transmitted from particle to particle. Probably this has been answered satisfactorily long ago by other experimenters; but this being a salt eminently fitted to determine this question, I made the following experiment:-Into one division of the decomposition cell, a solution of pure cyanide of potassium and silver was introduced, and into the other division a neutral solution of chloride of potassium. By this arrangement, I considered that, if the cyanogen travelled direct, cyanide of silver should be formed upon the positive electrode which was immersed in the chloride of potassium; and if it was transmitted from particle to particle, chloride of silver would be deposited upon the pole and cyanide of potassium formed in the solution. The experiment verified this last supposition; after a current had passed through the solution for nearly eight hours the positive electrode was completely encased in a horny crust of considerable flexibility, which was not soluble in strong boiling nitric acid nor in hot sulphuric acid, and did not give off fumes of hydrocyanic acid on the addition of muriatic acid. 25 grains gave 18.6 of silver, which with the previous experiments is sufficient to prove the substance to be chloride of silver; the solution had the smell of cyanide of potassium, which had dissolved or decomposed a portion of the chloride of silver it held in solution.

LXVI. On Sonorous Phanomena in Electro-Magnets. By J. P. Markian.

To the Editors of the Philosophical Magazine and Journal. Gentiemen,

HAVING noticed a few phænomena resulting from magnetic influence in bars of soft iron, and which I have not before heard of, I am induced to submit an account of 